\title{
A stochastic programming model for the optimal electricity market bid problem with bilateral contracts for thermal and combined cycle units*
}

\author{
F.-Javier Heredia, Marcos-J. Rider, Cristina Corchero ${ }^{\dagger}$ \\ GNOM (Group on Numerical Optimization and Modeling) \\ Statistics and Operations Research Department \\ Technical University of Catalonia \\ e-mail: \{f.javier.heredia, marcos.rider, cristina.corchero\}@upc.edu \\ E-prints UPC - http://hdl.handle.net/2117/2282
}

October 3, 2008

\begin{abstract}
This paper developed a stochastic programming model that integrated the most recent regulation rules of the Spanish peninsular system for bilateral contracts in the day-ahead optimal bid problem. Our model allows a price-taker generation company to decide the unit commitment of the thermal and combined cycle programming units, the economic dispatch of the bilateral contracts between all the programming units and the optimal sale bid by observing the Spanish peninsular regulation. The model was solved using real data of a typical generation company and a set of scenarios for the Spanish market price. The results are reported and analyzed.
\end{abstract}

Keywords: Electricity spot-market - Short-term - Combined cycle units - Bilateral contracts - Optimal bid - Stochastic programming

\section{Introduction}

Generation companies in liberalized electricity markets do not have a load of their own to satisfy, but must bid their hourly generation to the market operator, who selects the lowest-price among the biding companies to match the pool load. A specific generation company (GenCo) expects to have most of its bids accepted, i.e., have them priced below the market price, determined hourly by matching the lowest-price bids with the pool load. Liberalized electricity markets are nowadays very sophisticated energy- and financial-transaction multimarkets where, around the main electricity market, the so-called "day-ahead" or "spot" market, a portfolio of other financial and physical markets as well as bilateral contracts (BCs) exist. Moreover, a generation company operating in such a complex market can no longer optimize its medium- and short-term generation planning decisions without considering the relation between those markets and the increasing importance of the emission-free (wind power and hydro-generation) and low-emission technologies (combined cycle).

\footnotetext{
*This work was supported by the Ministry of Science and Technology of Spain through CICYT Project DPI200509117-C02-01 (http://www-eio.upc.es/research/gnom/meccyt06-08/).

${ }^{\dagger}$ Grant FPI BES-2006-12311 of the Ministry of Science and Technology of Spain.
} 
Table 1: Generation of electricity in the Spanish Peninsular Electricity System (GWh)

\begin{tabular}{lllllll}
\hline & 2002 & 2003 & 2004 & 2005 & 2006 & 2007 \\
\hline Hydro & 22.598 & 38.874 & 29.777 & 19.170 & 24.761 & 26.381 \\
Nuclear & 63.016 & 61.875 & 63.606 & 57.539 & 60.184 & 55.046 \\
Coal & 78.768 & 72.249 & 76.358 & 77.393 & 66.143 & 71.846 \\
Fuel & 16.474 & 8.027 & 7.697 & 10.013 & 5.841 & 2.384 \\
CC & 5.308 & 14.991 & 28.974 & 48.840 & 63.561 & 68.304 \\
\hline Subtotal & 186.164 & 196.016 & 206.412 & 212.955 & 220.490 & 223.962 \\
\hline Self-consumption & -8.420 & -8.162 & -8.649 & -9.080 & -8.719 & -8.655 \\
\hline Cogeneration & & & & & & \\
and renewables & 35.401 & 41.412 & 45.868 & 50.365 & 49.904 & 55.754 \\
\hline Total & 213.145 & 229.266 & 243.631 & 254.240 & 261.675 & 271.061 \\
\hline
\end{tabular}

The combined cycle (CC) units represent the majority of the new generating unit installations across the globe. Their advantages are: a) High efficiency (can reach 60\%, which is a $20-30 \%$ improvement over that of the traditional single-cycle thermal plants); b) Fast response (can be quite instrumental in facing rapid fluctuations in the power markets); c) Environmental friendliness (the $\mathrm{CO}_{2}$ production of a natural gas fueled $\mathrm{CC}$ plant is much lower than that of other fossil-fueled turbine technologies); and d) Compact and shorter installation time. For example, as of December 31, 2007, the total installed capacity in the Spanish Peninsular Electricity System was 85.959 MW (this capacity has increased by 26.140 MW since 2002). This increase is chiefly attributed to the commissioning of new CC, cogeneration, and renewable power plants, most of which comprised the wind power. Currently, the installed capacity of CC units represents $24.38 \%$ of the total installed capacity. Also, the total net generation in the Spanish Peninsular Electricity System reached 271 TWh (representing an increase of around 58 TWh over 2002), with 25\% from coal plants, $24 \%$ from CC plants, $21 \%$ from cogeneration and renewable plants, $20 \%$ from nuclear plants, $9 \%$ from hydro plants and $1 \%$ from fuel plants (Table 1);

The BCs are agreements between a generation company and a qualified consumer to provide a given amount of electrical energy at a stipulated price along with a delivering period. The characteristics of the BC (energy, price and delivering period) are negotiated before the day-ahead market, either in organized or non-organized markets. In the organized BC markets, the producers and consumers send sell and purchase bids that are matched by the market operator. Examples of organized BC markets are the CESUR (CESUR, 2008) and EPE (MEFF, 2008) auctions in Spain, BelpexVPP auctions in Belgium (BELPEX, 2008), and the EDF's capacity auctions in France (EDF, 2008). In non-organized BC markets, the producers and consumers agree the amount, price, and period of the energy delivered, in a private negotiation. In both cases, from the point of the view of the generation company, a BC represents a scheduled load curve to be delivered, chargeable at a fixed price.

Several researchers have proposed optimal bidding models in the day-ahead market for thermal units under the price-taker assumption, with or without BC. Some researchers (Conejo and Arroyo, 2002) presented a mixed-integer programming model to optimize the production schedule of a single unit with a simple bidding strategy. Furthermore, in another study (Gountis and Bakirtzis, 2004), the approximation of step-wise bid curves by linear bid functions, based on the marginal costs was considered, although in a context without BC. Also, in an earlier study (Ni et al, 2004), the concept of price-power function, which is similar to the matched energy function defined in our study, was used to derive the optimal bid curves of a hydro-thermal system under the assumption that the spot prices for the day-ahead and reserve markets behave as a Markov Chain. The mixed-integer stochastic programming model (Nowak et al, 2005) distinguishes the variables corresponding to the bid energy and those representing the matched energy, though in a price-maker framework and without BC. In another model (Shresta et al, 2004), very much related in some aspects to 


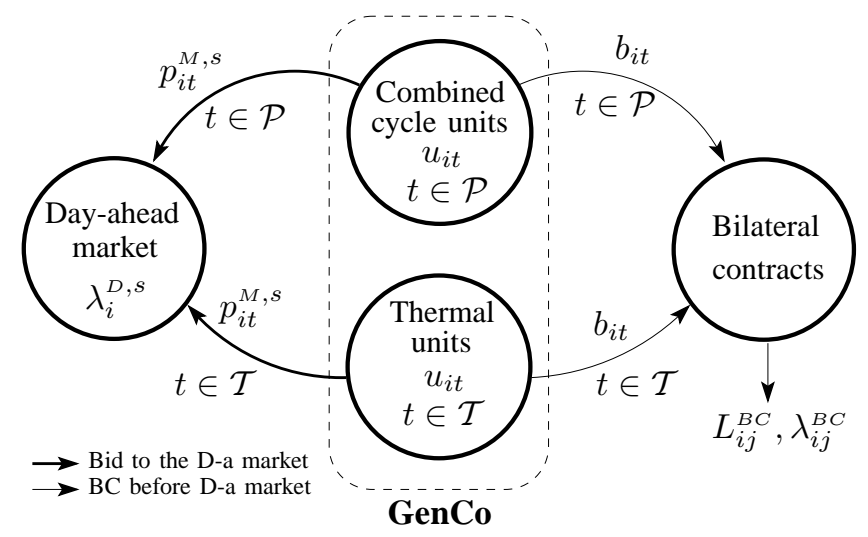

Figure 1: Representation of the system under study

the one presented in our study, a stochastic unit commitment problem with BC was solved by maximizing the day-ahead market benefit. The stochasticity in the spot prices was introduced through a set of scenarios, giving rise to a two-stage stochastic programming problem. In another earlier study (Triki et al, 2005), the researchers presented a mixed-integer stochastic optimization model for scheduling the thermal units, and the production plants were optimized in the presence of stochastic market-clearing prices. Nevertheless, the two earlier models (Shresta et al, 2004; Triki et al, 2005) failed to propose any explicit modelization of the optimal bid as we have done. Furthermore, the general considerations about the bidding process in electricity markets can also be obtained (Anderson and Philpott, 2002, 2003; Neame et al, 2003).

One of the earlier studies (Bjelogrlic, 2000) considered the CC units in the short-term resource scheduling. The proposed algorithm was based on the assumption that the thermal subsystem of a $\mathrm{CC}$ is modeled through input-output curves that are defined for all configurations and all steam load ranges. A method (Lu and Shahidehpour, 2004) was presented to calculate the unit commitment of CC units using dynamic programming and lagrangian relaxation applied to the security-constrained short-term scheduling problem. Furthermore, the price-based unit commitment problem based on the mixed-integer programming method for a generating company with thermal, CC, cascadedhydro, and pumped-storage units has also been presented (Li and Shahidehpour, 2005). None of the earlier publications presented an explicit formulation of the optimal sale bid of the CC units to the day-ahead market or any considerations about the BC.

Our study developed an stochastic mixed-integer quadratic programming model for a pricetaker GenCo with BC obligations to determine the optimal bidding strategy of a pool of thermal and $\mathrm{CC}$ programming units in the day-ahead electricity market. The model allows a price-taker GenCo to decide the unit commitment of its thermal and CC programming units, the economic dispatch of the BC among the programming units, and the optimal bid for thermal and CC units. The main contributions of this paper include: (a) a new model for the optimal bid function and matched energy for thermal and CC units, (b) a new and detailed mixed-integer formulation of the operation rules of the $\mathrm{CC}$ units and $(c)$ the joint optimization of all the above-mentioned factors together with the $\mathrm{BC}$ duties. The model was tested with real data of market prices and programming units of a GenCo operating in the Spanish electricity market (OMEL, 2008).

In Section 2 of this paper, the stochastic programming model for the BC-constrained optimal bid problem is presented, where the unit commitment and $\mathrm{BC}$ constraints are defined and the model for the BC-constrained optimal bid function and equivalent constraints are given. In Section 3, the stochastic processes involving day-ahead market clearing prices are characterized and a detailed case study is solved and analyzed. Finally, in Section 4 some relevant conclusions are drawn.

\section{The Stochastic Programming Model}

Fig. 1 represents a price-taker GenCo possessing a set $\mathcal{T}$ of thermal units (coal, nuclear, fuel) and a set $\mathcal{C}$ of $\mathrm{CC}$ units (a combustion turbine and a steam turbine). Both thermal and CC units bid 


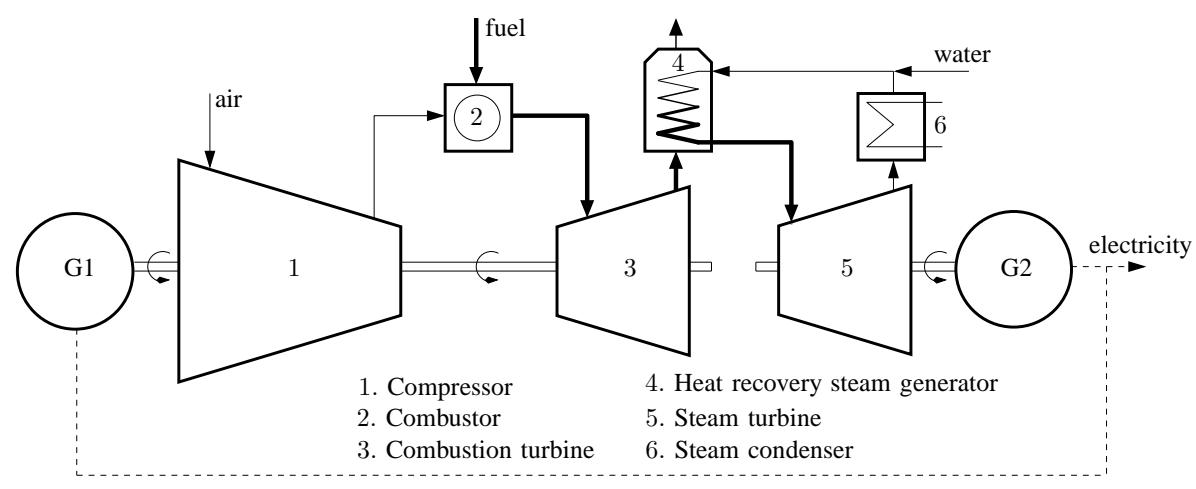

Figure 2: Combined cycle unit

to the $i \in \mathcal{I}=\{1,2, \ldots, 24\}$ hourly auctions of the day-ahead market (left-oriented arrows of Fig. 1). The stochasticity of the spot price $\lambda_{i}^{D}, i \in \mathcal{I}$ is represented by a set of $\mathcal{S}$ scenarios. The set $\mathcal{B C}$ represents the portfolio of BC duties, with known energy $\left(L_{i j}^{B C} \mathrm{MWh}\right)$ and price $\left(\lambda_{i j}^{B C} € / \mathrm{MWh}\right)$ for each $\mathrm{BC}$ contract $j \in \mathcal{B C}$ and time period $i \in \mathcal{I}$, that must be dispatched between the thermal and $\mathrm{CC}$ units (right-oriented arrows of Fig. 1). The main information provided by the model (here and now decisions or first-stage variables) for each period, $i \in \mathcal{I}$, are the unit commitment of the thermal and CC units (variables $u_{i t}$ ), the energy allocated to the portfolio of bilateral contracts by each thermal and $\mathrm{CC}$ units (variables $b_{i t}$ ), and the optimal sale bid, expressed as a function of the previous first-stage variables, which results in the second stage variables $p_{i t}^{M, s}$, the matched energy (Section 2.3).

\subsection{Thermal and Combined Cycle units operation}

In the traditional thermal units (single-cycle thermal plants), the fuel (natural gas) and the compressed air are mixed and burnt in a combustion chamber. The energy released during the combustion is used to turn a combustion turbine that drives an electric generator (G1) to produce electricity (Fig. 2). The air is a relatively non-problematic and inexpensive medium, which can be used in modern gas turbine. In the CC units, the heat captured from the exhaust gas of combustion turbine (CT) in the single cycle (which would otherwise be wasted), is used in the heat recovery steam generator (HRSG) that is used to turn a steam turbine (ST), which consequently drives an electric generator (G2) to produce additional electricity.

The CC units represent a combination of combustion and steam turbines within a power plant. Typically, a CC unit consists of several CTs and an HRSG/ST set. Based on the different combinations of CTs and HRSG/ST, a CC unit can operate at multiple states or configurations. The first two columns of Table 2 show the states of a CC unit with a CT and an HRSG/ST considered in this study. The operational rules of a typical CC unit were formulated ( $\mathrm{Lu}$ and Shahidehpour, 2004) with the help of the so-called pseudo units (PUs). As the thermal units, the PUs of each $\mathrm{CC}$ unit have their own unique cost characteristics, real power generation limits, minimum on time limits, etc., and can be viewed as a special set of non-independent or coupling single thermal units. Contrary to the model proposed earlier (Lu and Shahidehpour, 2004), where each one of the three states of the CC unit had its own PU, our formulation only considered two PUs, each associated with states 1 and 2 of the CC. The on/off state of these two PUs uniquely determined the state of the CC (see columns 3 and 5 of Table 2), and allowed (as it will be seen later) a correct modelization of the operation of the state 0 without the need of an additional PU.

Let us define $\mathcal{P}_{c}$, the set of PUs of the CC unit $c \in \mathcal{C}$, and $\mathcal{P}=\cup_{c \in \mathcal{C}} \mathcal{P}_{c}$, the complete set of PUs. By $\mathcal{P}_{c}(j)$, we denote the PU associated with the state $j \in\{1,2\}$ of the CC unit $c$. Thus, $\mathcal{U}=\mathcal{T} \cup \mathcal{P}$ represents the complete set of units (thermal and pseudo). The on/off state of each thermal and pseudo units at period $i$ can be represented by the first-stage binary variables $u_{i t}$, $t \in \mathcal{U}$. Columns 4 and 6 of Table 2 illustrate the relation of the commitment binary variables of the PUs, $u_{i \mathcal{P}_{c}(1)}$ and $u_{i \mathcal{P}_{c}(2)}$, with the state of the associated CC unit. 
Table 2: States of the CC unit and its associated Pseudo units

\begin{tabular}{|c|c|c|c|c|c|}
\hline \multicolumn{6}{|c|}{ CC unit with a CT and HRSG/ST } \\
\hline State & Composition & Pseudounit 1 & $u_{i \mathcal{P}_{c}(1)}$ & Pseudounit 2 & $u_{i \mathcal{P}_{c}(2)}$ \\
\hline 0 & 0CT+0HRSG/ST & off & 0 & off & 0 \\
\hline 1 & $1 \mathrm{CT}+0 \mathrm{HRSG} / \mathrm{ST}$ & on & 1 & off & 0 \\
\hline 2 & $1 \mathrm{CT}+1 \mathrm{HRSG} / \mathrm{ST}$ & off & 0 & on & 1 \\
\hline
\end{tabular}

However, the operation of each thermal unit must guarantee the minimum up $\left(t_{t}^{\text {on }}\right)$ and down $\left(t_{t}^{\text {off }}\right)$ times. These conditions are introduced through the following set of constraints (Nabona and Pages, 2007):

$$
\left.\begin{array}{l}
u_{i t}-u_{(i-1) t}-e_{i t}+a_{i t}=0 \quad(a) \\
a_{i t}+\sum_{j=i}^{\min \left\{i+t_{t}^{o f f},|\mathcal{I}|\right\}} e_{j t} \leq 1 \quad(b) \\
e_{i t}+\sum_{j=i+1}^{\min \left\{i+t_{t}^{o n},|\mathcal{I}|\right\}} a_{j t} \leq 1 \quad(c) \\
u_{i t}, a_{i t}, e_{i t} \in\{0,1\} \cap \mathcal{K}_{t}
\end{array}\right\} \quad \forall i \in \mathcal{I}, \forall t \in \mathcal{T}
$$

where Eq. (1a)-(1b) define the auxiliary binary variables $a_{i t}$ and $e_{i t}$ to be $a_{i t}=1$ iff $u_{(i-1) t}=1$ and $u_{i t}=0$, and $e_{i t}=1$ iff $u_{(i-1) t}=0$ and $u_{i t}=1$. The minimum up $\left(t_{t}^{o n}\right)$ and down $\left(t_{t}^{o f f}\right)$ times are guaranteed by Eq. (1b)-(1c). The set $\mathcal{K}_{t}$ stands for the initial state of each unit.

Analogously, each PU $t \in \mathcal{P}$ has its own minimum up time, $t_{t}^{o n}$ :

$$
\left.\begin{array}{l}
u_{i t}-u_{(i-1) t}-e_{i t}+a_{i t}=0 \quad(a) \\
e_{i t}+\sum_{j=i}^{\min \left\{i+t_{t}^{o n},|\mathcal{I}|\right\}} a_{j t} \leq 1 \quad(b) \\
u_{i t}, a_{i t}, e_{i t} \in\{0,1\} \cap \mathcal{K}_{t}
\end{array}\right\} \forall i \in \mathcal{I}, \forall t \in \mathcal{P}
$$

Each CC unit also has a minimum down time, i.e., once shut down, the CC unit cannot be started up before $t_{c}^{C}$ periods. Thus, we introduced the auxiliary variables $u_{i c}^{C}, a_{i c}^{C}$ and $e_{i c}^{C}$ to represent the on/off, shut-down, and start-up state, respectively, of the $\mathrm{CC}$ unit $c \in \mathcal{C}$. As in the case of the thermal and pseudo units, the following constraints formulate the minimum down time condition for the $\mathrm{CC}$ units:

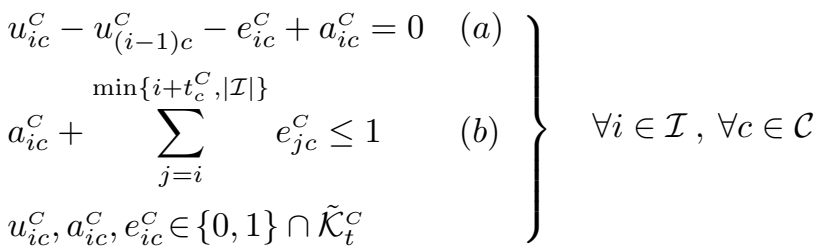

where set $\tilde{\mathcal{K}}_{t}^{C}$ stands for the initial state of each CC unit. In fact, variables $u_{i c}^{C}, a_{i c}^{C}$ and $e_{i c}^{C}$ are not necessary, because they can be expressed in terms of the binary variables of the PU of $\mathcal{P}_{c}$ with the aid of the feasible transition rules defined in Fig. 3:

$$
u_{i c}^{C}=u_{i \mathcal{P}_{c}(1)}+u_{i \mathcal{P}_{c}(2)} ; e_{i c}^{C}=e_{i \mathcal{P}_{c}(1)}-a_{i \mathcal{P}_{c}(2)} ; a_{i c}^{C}=a_{i \mathcal{P}_{c}(1)}-e_{i \mathcal{P}_{c}(2)}
$$

and, the constraints (3) can be re-expressed in terms of the PU variables as: 


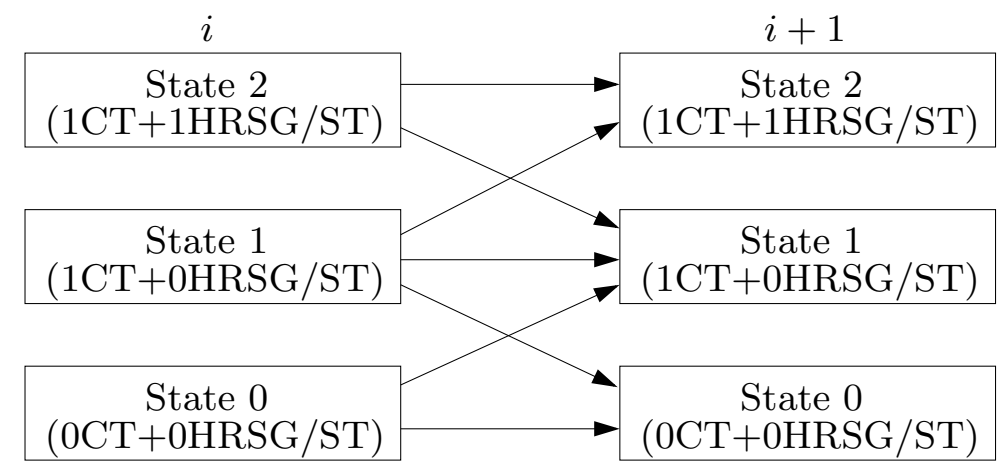

Figure 3: Feasible transitions of the CC unit with a CT and HRSG/ST

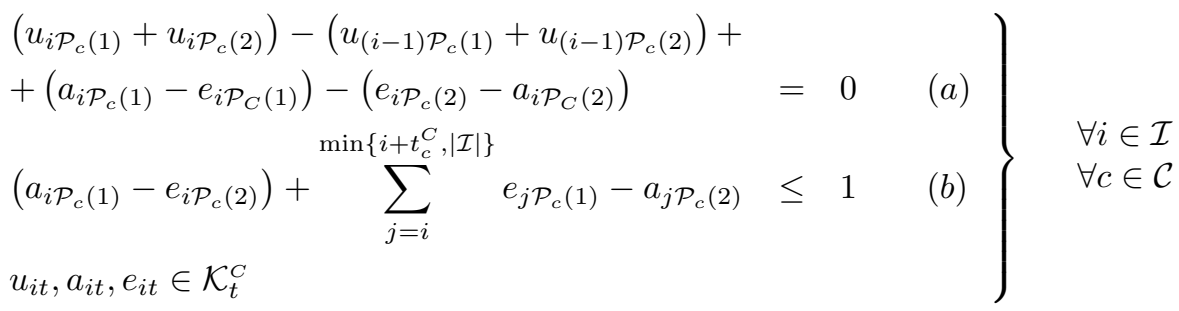

with $\mathcal{K}_{t}^{C}$ standing for conditions imposed by the initial state of the CC unit. The feasible transitions rules (Fig. 3) impose additional constraints to the operation of the PUs associated to the same $\mathrm{CC}$ unit, $c \in \mathcal{C}$. First, the PUs in $\mathcal{P}_{c}$ are mutually exclusive (Eq. $(6 a)$ ), i.e., only one of them can be committed at a given period (a CC can only be in one state simultaneously). Second, the change of the commitment of the PUs in $\mathcal{P}_{c}$ between periods $i$ and $i+1$ are limited to the feasible transitions depicted in Fig. 3. These feasible transitions impose that, if the $\mathrm{CC}$ unit $c$ is in state 0 at period $i\left(u_{i \mathcal{P}_{c}(1)}+u_{i \mathcal{P}_{c}(2)}=0\right)$, it cannot be in state 2 at period $i+1\left(u_{(i+1) \mathcal{P}_{c}(2)}=0\right)$ (Eq. $(6 b))$. Conversely, if $u_{i \mathcal{P}_{c}(2)}=1$, then $u_{(i+1) \mathcal{P}_{c}(1)}+u_{(i+1) \mathcal{P}_{c}(2)} \geq 1$ (Eq. $\left.(6 c)\right)$. The following set of constraints formulates the specific operation rules of the CC units:

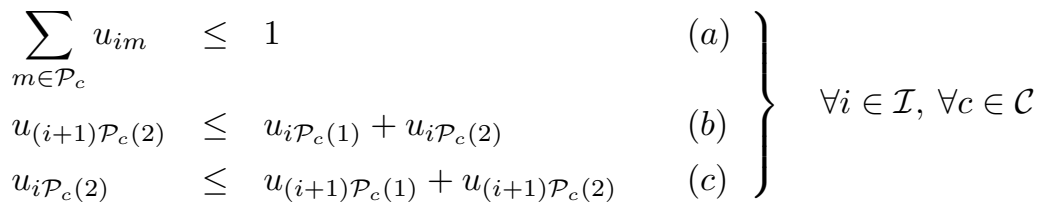

\subsection{Bilateral Contracts Constraint}

Consider that the GenCo has agreed to physically provide the energy amounts $L_{i j}^{B C}$ at hour $i \in \mathcal{I}$ of day $\mathrm{D}$ for each one of the $j \in \mathcal{B C}$ bilateral contracts. This energy $L_{i j}^{B C}$ can be provided by any programming unit $\mathcal{U}$, both thermal and PUs:

$$
\left.\begin{array}{l}
\sum_{t \in \mathcal{U}} b_{i t}=\sum_{j \in \mathcal{B C}} L_{i j}^{B C} \quad(a) \\
b_{i t} \in\left[0, \bar{p}_{t}\right] \quad \forall t \in \mathcal{U} \quad(b)
\end{array}\right\} \forall i \in \mathcal{I}
$$

where the total contribution of the committed unit $t$ to the BC covering at period $i$ is represented by the variable $b_{i t}$. The system of constraints $(1,2,5,6,7)$ defines the set $\Omega$

$$
\Omega=\left\{u \in\{0,1\}^{|\mathcal{I}| \times|\mathcal{U}|}, b \in \Re^{|\mathcal{I}| \times|\mathcal{U}|} \mid[u, b] \text { satisfies }(1,2,5,6,7)\right\}
$$

of all the feasible unit commitment and BC-dispatching solutions $[u, b]$. In the following section the optimal sale bid for each feasible policy $[u, b]$ is developed and the expression of the associated matched energy is formulated. 


\subsection{Optimal bid function and equivalent matched energy constraints}

In the MIBEL, a simple sale bid of unit $t$ for the $i$ th day-ahead market consists on a step-wise non-decreasing curve defined with up to 10 power(MWh)/price $(€ / \mathrm{MWh})$ blocks

$$
\left(p_{i t k}^{B}, \lambda_{i t k}^{B}\right), k=1, \ldots, 10
$$

However, after the market clearing all the blocks with a bid price $\lambda_{i t k}^{B}$ not greater than the clearing price $\lambda_{i}^{D}$ will be accepted (matched), which implies that the matched energy

$$
p_{i t}^{M}\left(\lambda_{i}^{D}\right)=\sum_{k \mid \lambda_{i t k}^{B} \leq \lambda_{i}^{D}} p_{i t k}^{B}
$$

should be generated and rewarded at price $\lambda_{i}^{D}$, and the rest blocks of Eq. (9) will be neglected. The MIBEL's rules state that each GenCo must notify the scheduling of the BCs (first-stage variables $b_{i t}$ ) to the system and market operator, at the most 25 minutes before the closure of the day-ahead market. This implies that, at the point of the decision-making procedure where the GenCo must decide its optimal bid (9), the scheduling variables of BC's, $b_{i t}$, are fixed and can be viewed as constant parameters. Subsequently, in this situation, the problem that the GenCo faces is that of maximizing its day-ahead market's benefit for a given BC's dispatched energy. The objective of this section is to develop the optimal bid function, $\lambda_{i t}^{B *}\left(p_{i t}^{B}\right)$, that gives the optimal bid price, $\lambda_{i t}^{B}$, at which the capacity generation, $p_{i t}^{B}$ must be bid to maximize the benefit from the pool for a given BC's dispatched-energy $b_{i t}$. To ease the modelization, we considered $\lambda_{i t}^{B *}\left(p_{i t}^{B}\right)$ as a general function, not necessarily step-wise. This is a common simplified representation of the true sale bid (9) used by several authors (e.g., (Gountis and Bakirtzis, 2004)). Furthermore, it is always possible to adapt a posteriori the resulting optimal bid function to the step-wise representation (9).

The optimal bid function could be developed under the the following assumptions:

Assumption 1. The GenCo is a price-taker, i.e., the day-ahead clearing price $\lambda_{i}^{D}$ does not depend on the GenCo's bidding.

Assumption 2. The unit $t$ (thermal or PU) has been committed $\left(u_{i t}=1\right)$.

Assumption 3. To guarantee the commitment of the unit $t$ in the operational programming resulting from the clearing of the day-ahead market, the unit $t$ would bid its minimum generation output $\underline{p}_{t}$ at zero price (instrumental bid).

Assumption 4. To respect the MIBEL rules, the total contribution of unit to the BC covering must be excluded from the bid.

Assumption 5. The probability function of the clearing-price random variable $\lambda_{i}^{D}$ has been discretized in a set of scenarios $\mathcal{S}$ with associated clearing price $\lambda_{i}^{D, s}$ and probability $P^{s}, s \in \mathcal{S}$.

Assumption 4 implies that the non-negative bid energy $p_{i t}^{B}$ must be upper bounded by $\left(\bar{p}_{t}-b_{i t}\right)$. By assuming the quadratic thermal generation costs, $C^{G}(p)=c_{t}^{b}+c_{t}^{l} p+c_{t}^{q}(p)^{2}$, the benefits obtained from the day-ahead market as a function of the matched energy $p_{i t}^{M, s}$ (the amount of energy that the thermal unit $t$ has to produce as a result of the market-clearing mechanism) for a given dispatched BC energy $b_{i t}$, under scenario $s$ will be:

$$
\begin{aligned}
B_{i t}^{D, s}\left(p_{i t}^{M, s}, b_{i t}\right) & =\lambda_{i}^{D, s} p_{i t}^{M, s}-\left[C^{G}\left(p_{i t}^{M, s}+b_{i t}\right)-C^{G}\left(b_{i t}\right)\right] \\
& =\lambda_{i}^{D, s} p_{i t}^{M, s}-c_{t}^{l} p_{i t}^{M, s}-c_{t}^{q}\left(p_{i t}^{M, s}\right)^{2}-2 c_{t}^{q} p_{i t}^{M, s} b_{i t}
\end{aligned}
$$

The expression of the optimal bid function is established by the following theorem:

Theorem 1. If assumptions 1-4 hold true, then the expression:

$$
\lambda_{i t}^{B *}\left(p_{i t}^{B}, b_{i t}\right)=\left\{\begin{array}{ll}
0 & \text { if } 0 \leq p_{i t}^{B} \leq\left[\underline{p}_{t}-b_{i t}\right]^{+} \\
2 c_{t}^{q}\left(p_{i t}^{B}+b_{i t}\right)+c_{t}^{l} & \text { if }\left[\underline{p}_{t}-b_{i t}\right]^{+}<p_{i t}^{B} \leq \bar{p}_{t}-b_{i t}
\end{array} \quad \forall i \in \mathcal{I}, \forall t \in \mathcal{U}\right.
$$

with $[a-b]^{+}=\max \{0, a-b\}$, is the optimal bid function of unit $t$ for the day-ahead market $i$ in the sense that, for any given value $b_{i t}$, if function (12) is bid, the matched energy $p_{i t}^{M, s}$ corresponding to any scenario $s$ with market price $\lambda_{i}^{D, s}$, maximizes the day-ahead benefit function (11) 


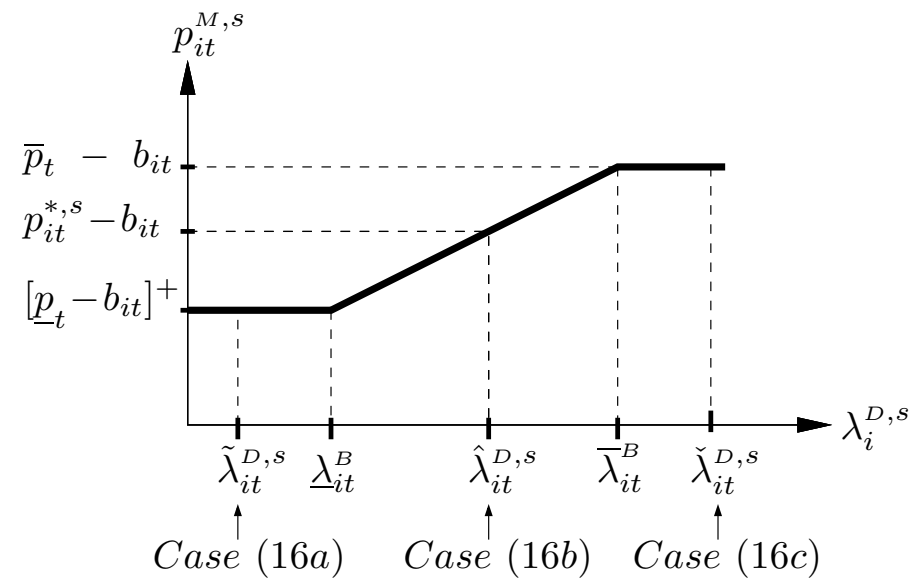

Figure 4: Matched bid energy function $p_{i t}^{M, s}$ as a function of the clearing market price $\lambda_{i}^{D, s}$ corresponding to the optimal bid function (12)

Proof. The first block of the optimal bid function $\lambda_{i t}^{B *}\left(p_{i t}^{B}, b_{i t}\right)=0$ for $p_{i t}^{B} \leq\left[\underline{p}_{t}-b_{i t}\right]^{+}$is the instrumental bid needed to guarantee assumptions 3 and 4 .

To observe the optimality of the second part of the bid function, we must maximize the dayahead benefit function (11) with respect to the matched energy $p_{i t}^{M, s}$ into the interval:

$$
\left.\left.p_{i t}^{M . s} \in\right]\left[\underline{p}_{t}-b_{i t}\right]^{+}, \bar{p}_{t}-b_{i t}\right]
$$

The matched energy that maximizes the day-ahead benefit function can be easily defined as:

$$
p_{i t}^{M, s}\left(b_{i t}\right)=\operatorname{argsup}_{p_{i t}^{M, s}}\left\{B_{i t}^{D, s}\left(p_{i t}^{M, s}, b_{i t}\right) \quad \mid \quad\left[\underline{p}_{t}-b_{i t}\right]^{+}<p_{i t}^{M, s} \leq \bar{p}_{t}-b_{i t}\right\}
$$

which can be obtained using:

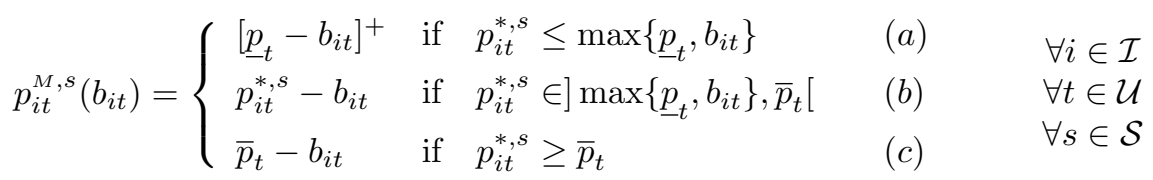

where $p_{i t}^{*, s}=\left(\lambda_{i}^{D, s}-c_{t}^{l}\right) / 2 c_{t}^{q}$, i.e., the unconstrained maximum of the benefit function (11) when $b_{i t}=0$, is a constant parameter that only depends on the scenario, period and unit.

Let us now analyze the expression of the matched energy associated with the bid (12) for all the possible values of the clearing market price $\lambda_{i}^{D, s}$. The following three cases can be distinguished (see Fig 4):

$$
\begin{cases}\lambda_{i}^{D, s} \leq \underline{\lambda}_{i t}^{B} & (a) \\ \left.\lambda_{i}^{D, s} \in\right] \underline{\lambda}_{i t}^{B}, \bar{\lambda}_{i t}^{B}[\quad(b) & (c) \\ \lambda_{i}^{D, s} \geq \bar{\lambda}_{i t}^{B}\end{cases}
$$

where $\underline{\lambda}_{i t}^{B}$ and $\bar{\lambda}_{i t}^{B}$ are the threshold prices:

$$
\underline{\lambda}_{i t}^{B}=2 c_{t}^{q}\left(\left[\underline{p}_{t}-b_{i t}\right]^{+}+b_{i t}\right)+c_{t}^{l} \quad ; \quad \bar{\lambda}_{i t}^{B}=2 c_{t}^{q} \bar{p}_{t}+c_{t}^{l}
$$

It can be easily observed that cases $(15 a),(b)$, and $(c)$ are equivalent to cases $(14 a),(b)$, and (c), respectively,i.e.,

$$
\begin{aligned}
\lambda_{i}^{D, s} \leq \underline{\lambda}_{i t}^{B} & \Longleftrightarrow p_{i t}^{*, s} \leq \max \left\{\underline{p}_{t}, b_{i t}\right\} \\
\left.\lambda_{i}^{D, s} \in\right] \underline{\lambda}_{i t}^{B}, \bar{\lambda}_{i t}^{B}[ & \left.\Longleftrightarrow p_{i t}^{*, s} \in\right] \max \left\{\underline{p}_{t}, b_{i t}\right\}, \bar{p}_{t}[ \\
\lambda_{i}^{D, s} \geq \bar{\lambda}_{i t}^{B} & \Longleftrightarrow p_{i t}^{*, s} \geq \bar{p}_{t}
\end{aligned}
$$


Let us now observe the expression of the matched energy associated with the three cases $(15 a)$, $(b)$, and $(c)$, which also coincides with the optimal matched energy, $p_{i t}^{M, s}$, for the three cases of Eq. (14):

Case (15a) : If the market clearing price $\lambda_{i}^{D, s}$ is below the minimum non-instrumental bid price $\underline{\lambda}_{i t}^{B}$ (see $\tilde{\lambda}_{i t}^{D, s}$ in Fig. 4), then only the instrumental part of the bid (12) is accepted and the matched energy will be $\left[\underline{p}_{t}-b_{i t}\right]^{+}$,i.e, the same amount as in case $(14 a)$.

Case $(\mathbf{1 5} b)$ : When the market clearing price $\lambda_{i}^{D, s}$ is strictly between the threshold prices (see $\hat{\lambda}_{i t}^{D, s}$ in Fig. 4), only the bid energy with a bid price less than or equal to this clearing price will be accepted (matched). The matched energy obtained from the expression of the optimal bid function in this case is $\left(\lambda_{i}^{D, s}-c_{t}^{l}\right) / 2 c_{t}^{q}-b_{i t}$, which is the same expression as in case $(14 b)$.

Case (15c) : If the market clearing price $\lambda_{i}^{D, s}$ is above the maximum bid price $\bar{\lambda}_{i t}^{B}$ (see $\breve{\lambda}_{i t}^{D, s}$ in Fig. 4)then the maximum generation bid $\left(\bar{p}_{t}-b_{i t}\right)$ is matched, which is the same amount as in case $(14 c)$.

Subsequently, it has been proved that if the proposed function (12) is bid to the day-ahead market, then the resulting matched energy will maximize the day-ahead benefit function (11)

When $b_{i t}=0$ (the committed unit $t$ does not contribute to the BC covering), our problem reduces to the classical self-commitment problem discussed by several authors (Gountis and Bakirtzis, 2004; Conejo and Arroyo, 2002). In this case, it is well known that the optimal-bid strategy for a price-taker GenCo consists of an instrumental bid up to the operational minimum limit, $\underline{p}_{t}$, and the rest of the plant capacity at the marginal price, $2 c_{t}^{q} p_{i t}+c_{t}^{l}$. This optimal policy corresponds to the particular case $b_{i t}=0$ of the generalized optimal-bid function (12). The matched energy for this particular case will be called the BC-free day-ahead matched energy, and can be represented by $p_{i t}^{D, s}$ :

$$
p_{i t}^{D, s} \equiv p_{i t}^{M, s}(0)=\left\{\begin{array}{llll}
\underline{p}_{t} & \text { if } \quad p_{i t}^{*, s} \leq \underline{p}_{t} & (a) & \forall i \in \mathcal{I} \\
p_{i t}^{*, s} & \text { if } \left.p_{i t}^{*, s} \in\right] \underline{p}_{t}, \bar{p}_{t}[ & (b) & \forall t \in \mathcal{U} \\
\bar{p}_{t} & \text { if } p_{i t}^{*, s} \geq \bar{p}_{t} & (c) & \forall s \in \mathcal{S}
\end{array}\right.
$$

where $p_{i t}^{D, s}$ is a constant parameter of the model for a fixed thermal $t$, period $i$, and scenario $s$, and can be used to develop a simplified expression of the optimal matched energy $p_{i t}^{M, s}\left(b_{i t}\right)$.

Proposition 1. The optimal matched energy function (14) can be expressed as:

$$
p_{i t}^{M, s}\left(b_{i t}\right)=\left[p_{i t}^{D, s}-b_{i t}\right]^{+} \quad \forall i \in \mathcal{I}, \forall t \in \mathcal{U}, \forall s \in \mathcal{S}
$$

with the constant parameter $p_{i t}^{D, s}$ defined in Eq. (19).

Proof. To observe the equivalence of Eq. (14) and Eq. (20), the three cases $(14 a)$, (b), and (c) can be analyzed:

Case (14a): This is the case where $p_{i t}^{*, s} \leq \max \left\{\underline{p}_{t}, b_{i t}\right\}$. If $\max \left\{\underline{p}_{t}, b_{i t}\right\}=\underline{p}_{t}$ then,

$$
p_{i t}^{*, s} \leq \underline{p}_{t} \Rightarrow p_{i t}^{D, s}=\underline{p}_{t} \Rightarrow\left[p_{i t}^{D, s}-b_{i t}\right]^{+}=\left[\underline{p}_{t}-b_{i t}\right]^{+}=p_{i t}^{M, s}\left(b_{i t}\right)
$$

Conversely, if $\max \left\{\underline{p}_{t}, b_{i t}\right\}=b_{i t}$, then either $p_{i t}^{*, s} \leq \underline{p}_{t}$, which has just been analyzed, or $\underline{p}_{t}<p_{i t}^{*, s} \leq b_{i t}$. In this last case, as $b_{i t} \leq \bar{p}_{t}$, Eq. (19) sets $p_{i t}^{D, s}=p_{i t}^{*, s}$, and both $\left[p_{i t}^{D, s}-b_{i t}\right]^{+}$ and $\left[p_{t}-b_{i t}\right]^{+}$take the value of zero.

Case $(\mathbf{1 4 b}):$ In this case, $\left.p_{i t}^{*, s} \in\right] \underline{p}_{t}, \bar{p}_{t}\left[\right.$ and through Eq. (19), $p_{i t}^{D, s}=p_{i t}^{*, s}$. The matched energy is:

$$
\left[p_{i t}^{D, s}-b_{i t}\right]^{+}=\left[p_{i t}^{*, s}-b_{i t}\right]^{+}=\left|p_{i t}^{*, s}>b_{i t}\right|=p_{i t}^{*, s}-b_{i t}=p_{i t}^{M, s}\left(b_{i t}\right)
$$

Case (14c) : Finally, when $p_{i t}^{*, s} \geq \bar{p}_{t}$, Eq. (19) sets $p_{i t}^{D, s}=\bar{p}_{t}$, and consequently,

$$
\left[p_{i t}^{D, s}-b_{i t}\right]^{+}=\left[\bar{p}_{t}-b_{i t}\right]^{+}=\left|b_{i t} \leq \bar{p}_{t}\right|=\bar{p}-b_{i t}=p_{i t}^{M, s}\left(b_{i t}\right)
$$




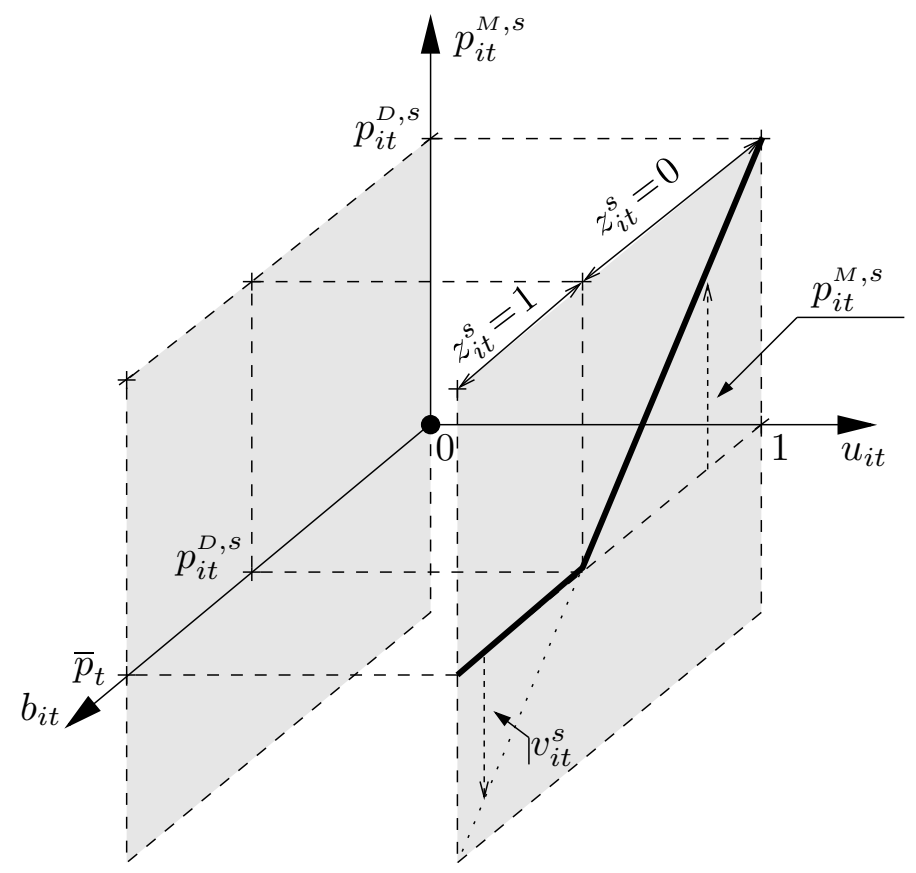

Figure 5: The matched energy function $p_{i t}^{M, s}$ for a fixed spot price $\lambda_{i}^{D, s}$

Proposition 1 sets that for a committed unit $t\left(u_{i t}=1\right)$ that bids the function (12) to the $i$ th day-ahead market, the matched energy at scenario $s$ will be $\left[p_{i t}^{D, s}-b_{i t}\right]^{+}$. However, if the unit is uncommitted $\left(u_{i t}=0\right)$, then the bid does not exist and the matched energy becomes zero. The matched energy can be then expressed through the matched energy function as a function of variables $b_{i t}$ and $u_{i t}$ :

$$
p_{i t}^{M, s}\left(b_{i t}, u_{i t}\right)=\left\{\begin{array}{ll}
{\left[p_{i t}^{D, s}-b_{i t}\right]^{+}} & \text {if } u_{i t}=1 \\
0 & \text { if } u_{i t}=0
\end{array} \quad \forall i \in \mathcal{I}, \forall t \in \mathcal{U}, \forall s \in \mathcal{S}\right.
$$

Fig. 5 represents the function $p_{i t}^{M, s}\left(b_{i t}, u_{i t}\right)$ (thick line and dot). However, this non-differentiable expression cannot be included in the optimization model as it is. To formulate an equivalent mixedinteger linear formulation, we introduced the auxiliary binary $z_{i t}^{s}$ and continuous $v_{i t}^{s}$ variables (see Fig 5). In this formulation, $z_{i t}^{s}=1$ whenever $b_{i t} \geq p_{i t}^{D, s}$ and $z_{i t}^{s}=0$ otherwise, while $v_{i t}^{s}$ will be always defined as $v_{i t}^{s}=\left[b_{i t}-p_{i t}^{D, s}\right]^{+}$. With the help of these auxiliary variables, expression (24) can be transformed into the following equivalent mixed-integer linear system:

$$
\begin{array}{ll}
p_{i t}^{M, s}=p_{i t}^{D, s} u_{i t}+v_{i t}^{s}-b_{i t} & (a) \\
p_{i t}^{D, s}\left(z_{i t}^{s}+u_{i t}-1\right) \leq b_{i t} & (b) \\
b_{i t} \leq p_{i t}^{D, s}\left(1-z_{i t}^{s}\right)+\bar{p}_{t}\left(z_{i t}^{s}+u_{i t}-1\right) & (c) \\
p_{i t}^{D, s}\left(1-z_{i t}^{s}\right) \geq p_{i t}^{M, s} & (d) \\
p_{i t}^{D, s}\left(1-z_{i t}^{s}\right) \leq p_{i t}^{D, s} u_{i t} & (e) \\
v_{i t}^{s} \leq\left(\bar{p}_{t}-p_{i t}^{D, s}\right)\left(z_{i t}^{s}+u_{i t}-1\right) & (f) \\
p_{i t}^{M, s} \in\left[0, p_{i t}^{D, s}\right] & (g) \\
v_{i t}^{s} \in\left[0, \bar{p}_{t}-p_{i t}^{D, s}\right] & \forall t \in \mathcal{I} \\
z_{i t}^{s} \in\{0,1\} & (h) \\
&
\end{array}
$$


The following proposition establishes the equivalence between the function (24) and the system (25) over the set $\Omega$ of all the feasible unit commitment and BC-dispatching solutions:

Proposition 2. The system (25) and the function (24) are equivalent in the sense that for every feasible solution, $[u, b] \in \Omega$, the value of the matched energy variable $p_{i t}^{M, s}$ at every feasible solution of the system (25) satisfies function (24).

Proof. First, let us consider the solution of system (25) for all the feasible solutions in $\Omega$ with $u_{i t}=0$. As the parameter $p_{i t}^{D, s}$ is always non-negative, by Eq. (25e), $z_{i t}^{s}=1$, and Eq. (25d), together with the bounds of Eq. $(25 g)$ sets $p_{i t}^{M, s}=0$. Analogously, Eq. (25c) and Eq. (25f), together with the bounds Eq. (7b) and Eq. (25h) zeroes the values of $b_{i t}$ and $v_{i t}^{s}$, respectively. The remaining equations, Eq. $(25 a)$ and Eq. (25b), result in the redundant relations $p_{i t}^{M, s}=0$ and $b_{i t} \geq 0$, respectively.

Second, let us analyze system (25) for all the feasible solutions in $\Omega$ with $u_{i t}=1$. For all these solutions, system $(24)$ reduces to:

$$
\left.\begin{array}{ll}
p_{i t}^{M, s}=p_{i t}^{D, s}+v_{i t}^{s}-b_{i t} & (a) \\
p_{i t}^{D, s} z_{i t}^{s} \leq b_{i t} & (b) \\
b_{i t} \leq p_{i t}^{D, s}\left(1-z_{i t}^{s}\right)+\bar{p}_{t} z_{i t}^{s} & (c) \\
p_{i t}^{D, s}\left(1-z_{i t}^{s}\right) \geq p_{i t}^{M, s} & (d) \\
p_{i t}^{D, s}\left(1-z_{i t}^{s}\right) \leq p_{i t}^{D, s} & (e) \\
v_{i t}^{s} \leq\left(\bar{p}_{t}-p_{i t}^{D, s}\right) z_{i t}^{s} & (f) \\
p_{i t}^{M, s} \in\left[0, p_{i t}^{D, s}\right] & (g) \\
v_{i t}^{s} \in\left[0, \bar{p}_{t}-p_{i t}^{D, s}\right] & \forall i \in \mathcal{I} \\
z_{i t}^{s} \in\{0,1\} & \forall t \in \mathcal{U} \\
&
\end{array}\right\}
$$

The set of the feasible solutions of $\Omega$ with $u_{i t}=1$ can be partitioned depending on the value of variable $b_{i t}$. For those solutions in $\Omega$ with $u_{i t}=1$ and $b_{i t} \leq p_{i t}^{D, s}$, Eq. $(26 b)$ sets $z_{i t}^{s}=0$. Subsequently, Eq. (26f) together with the bounds $(26 h)$ sets $v_{i t}^{s} \leq 0$, and by Eq. $(26 a), p_{i t}^{M, s}=$ $p_{i t}^{D, s}-b_{i t}$, which coincides with the value given by the function (24) for $u_{i t}=1$ and $b_{i t} \leq p_{i t}^{D, s}$. Equations $(26 c),(26 d)$, and (26e) derive redundant expressions. Conversely, for those solutions in $\Omega$ with $u_{i t}=1$ and $b_{i t}>p_{i t}^{D, s}$, Eq. (26c) sets $z_{i t}^{s}=1$. Subsequently, Eq. (26d), together with the lower bound defined in Eq. $(26 g)$, sets $p_{i t}^{M, s}=0$, accordingly with the value of the matched energy defined by function (24). The remaining equations (26a), (26b), (26e), and (26f) provide redundant constraints.

Proposition 2 assures that: (a) every feasible solution of $\Omega$ satisfies the equivalent matchedenergy constraints $(25)$ and $(b)$ the value of variable $p_{i t}^{M, s}$ represents the true value of the matched energy function (24). Finally, modelization of the thermal units and PUs must include the following set of constraints that define the total generation output of thermal unit $t$ at each time period $i$ and scenario $s$ :

$$
p_{i t}^{s}=p_{i t}^{M, s}+b_{i t} \quad \forall i \in \mathcal{I}, \forall t \in \mathcal{U}, \forall s \in \mathcal{S}
$$

\subsection{Objective function}

The expected value of the benefit function $B$ can be expressed as: 


$$
\begin{aligned}
E_{\lambda^{D}} & {\left[B\left(u, a, e, p, p^{M} ; \lambda_{i}^{D}\right)\right]=} \\
& \sum_{\forall i \in \mathcal{I}} \sum_{\forall j \in \mathcal{B C}} \lambda_{i j}^{B C} L_{i j}^{B C} \\
& -\sum_{\forall i \in \mathcal{I}} \sum_{\forall t \in \mathcal{T}}\left[c_{t}^{o n} e_{i t}+c_{t}^{o f f} a_{i t}+c_{t}^{b} u_{i t}\right] \\
& -\sum_{\forall i \in \mathcal{I}} \sum_{\forall c \in \mathcal{C}}\left[c_{\mathcal{P}_{c}(1)}^{o n}\left(e_{i \mathcal{P}_{c}(1)}-a_{i \mathcal{P}_{c}(2)}\right)+c_{\mathcal{P}_{c}(2)}^{o n} e_{i \mathcal{P}_{c}(2)}+\sum_{\forall t \in \mathcal{P}_{c}} c_{t}^{b} u_{i t}\right] \\
& +\sum_{\forall i \in \mathcal{I}} \sum_{\forall t \in \mathcal{U}} \sum_{\forall s \in \mathcal{S}} P^{s}\left[\lambda_{i}^{D, s} p_{i t}^{M, s}-c_{t}^{l} p_{i t}^{s}-c_{t}^{q}\left(p_{i t}^{s}\right)^{2}\right]
\end{aligned}
$$

The term (28) is constant and corresponds to the BC profit. The term (29) is the on/off fixed cost of the unit commitment of the thermal units. The CC's start-up and fixed generation costs are formulated in term (30). In this formulation, as in ( $\mathrm{Lu}$ and Shahidehpour, 2004), only start-up costs are associated to the PU, and no cost is associated to the transition from state 2 to state 1. Both terms (29) and (30) are deterministics and does not depend on the realization of the random variable $\lambda^{D}$. Finally, expression (31) represents the expected value of the benefit from the day-ahead market for thermal and CC units, where $P^{s}$ is the probability of scenario $s$. Usually, the generation cost functions of the PUs are represented as piece-wise linear functions, but in this study, they were modeled as quadratic functions as done in a couple of earlier studies (Lu and Shahidehpour, 2004; Li and Shahidehpour, 2005). All the functions appearing in Eqs. (29) and (31) are linear except the generation costs in Eq. (31), which are concave quadratic $\left(c_{t}^{q} \geq 0\right.$, see Tables 3 and 4 ).

\subsection{Final model}

The final model developed in the previous sections is as follows:

$$
\left\{\begin{array}{lll}
\max & E_{\lambda^{D}}\left[B\left(u, a, e, p, p^{M} ; \lambda^{D}\right)\right] & \\
\text { s.t. : } & & \\
& \text { Eq. }(1) & \text { Thermal unit commitment constraints } \\
\text { Eq. }(2,5,6) & \text { Combined cycle unit commitment const. } \\
\text { Eq. }(7) & \text { Bilateral contracts dispatching const. } \\
\text { Eq. }(25) & \text { Optimal matched energy equivalent const. } \\
\text { Eq. }(27) & \text { Definition of the total unit's generation } p_{i t}^{s}
\end{array}\right.
$$

The deterministic equivalent of the two-stage stochastic problem (32) is a mixed, continuous-binary concave quadratic maximization problem with linear constraints that can be solved with the help of standard optimization software, as illustrated in the following section.

\section{$3 \quad$ Test and Results}

The model (32) has been tested using real data of a typical generation company and market prices for the Spanish Peninsular Electricity System (OMEL, 2008) and the results are reported in this section. The day under study is Monday, May 05 2008, in the electricity market of mainland Spain. 3 bilateral contracts, 4 thermal units, 2 combined cycle units with a CT and a HRSG/ST and 24 hours of study were used in the tests. The characteristics of the thermal and CC units and BCs are shown in Table 3, 4 and 5, respectively. The parameter $s t_{t}^{0}$ stands for the number of hours the unit has been on $\left(s t_{t}^{0}>0\right)$ or off $\left(s t_{t}^{0}<0\right)$ previous to the first optimization period. $t_{t}^{o n}=t_{t}^{o f f}=3 h$ for all the thermal units. The minimum off time for both $\mathrm{CC}$ units (parameter $t_{c}^{C}$ in Eq. 3) was set to 3 hours, and also both CC units was considered shut-down for 3 hours previous to the first optimization period. The model (32) has been implemented in AMPL (Fourer et al, 2003) and 
Table 3: Operational Characteristics of the Thermal Units

\begin{tabular}{lllllllll}
\hline$t$ & $\begin{array}{c}c_{t}^{b} \\
€\end{array}$ & $\begin{array}{c}c_{t}^{l} \\
€ / \mathrm{MWh}\end{array}$ & $\begin{array}{l}c_{t}^{q} \\
€ / \mathrm{MWh}^{2}\end{array}$ & $\begin{array}{l}\underline{p}_{t} \\
\mathrm{MW}\end{array}$ & $\begin{array}{l}\bar{p}_{t} \\
\mathrm{MW}\end{array}$ & $\begin{array}{l}s t_{t}^{0} \\
\mathrm{hr}\end{array}$ & $\begin{array}{l}c_{t}^{\text {on }} \\
€\end{array}$ & $\begin{array}{l}c_{t}^{\text {off }} \\
€\end{array}$ \\
\hline 1 & 151.08 & 40.37 & 0.015 & 160.0 & 350.0 & +3 & 412.80 & 412.80 \\
2 & 554.21 & 36.50 & 0.023 & 250.0 & 563.2 & +3 & 803.75 & 803.75 \\
3 & 327.02 & 28.85 & 0.036 & 160.0 & 370.7 & -2 & 438.40 & 438.40 \\
4 & 197.93 & 36.91 & 0.020 & 160.0 & 364.1 & -1 & 419.20 & 419.20 \\
\hline
\end{tabular}

Table 4: Operational Characteristics of the Combined Cycle Units

\begin{tabular}{llllllllll}
\hline$c$ & $\mathcal{P}_{c}$ & $\begin{array}{c}c_{t}^{b} \\
€\end{array}$ & $\begin{array}{l}c_{t}^{l} \\
€ / \mathrm{MWh}\end{array}$ & $\begin{array}{l}c_{t}^{q} \\
€ / \mathrm{MWh}^{2}\end{array}$ & $\begin{array}{l}\underline{p}_{t} \\
\mathrm{MW}\end{array}$ & $\begin{array}{l}\bar{p}_{t} \\
\mathrm{MW}\end{array}$ & $\begin{array}{l}s t_{t}^{0} \\
\mathrm{hr}\end{array}$ & $\begin{array}{l}c_{t}^{\text {on }} \\
€\end{array}$ & $\begin{array}{l}t_{t}^{\text {on }} \\
\mathrm{hr}\end{array}$ \\
\hline 1 & 5 & 151.08 & 50.37 & 0.023 & 160.0 & 350.0 & -2 & 803.75 & 2 \\
1 & 6 & 224.21 & 32.50 & 0.035 & 250.0 & 563.2 & -2 & 412.80 & 2 \\
2 & 7 & 163.11 & 55.58 & 0.019 & 90.0 & 350.0 & -2 & 320.50 & 2 \\
2 & 8 & 245.32 & 31.10 & 0.022 & 220.0 & 700.0 & -2 & 510.83 & 2 \\
\hline
\end{tabular}

Table 5: Characteristics of the Bilateral Contracts

\begin{tabular}{lll}
\hline$j$ & $\begin{array}{l}L_{j=1 \ldots 24}^{B C} \\
\text { MW }\end{array}$ & $\begin{array}{l}\lambda_{j=1 \ldots 24}^{B C} \\
€ / \mathrm{MWh}\end{array}$ \\
\hline 1 & 200 & 75 \\
2 & 150 & 73 \\
3 & 250 & 78 \\
\hline
\end{tabular}


Table 6: Optimization Characteristics of the Study Case

\begin{tabular}{lllll}
\hline Constraints & $\begin{array}{l}\text { Real } \\
\text { variables }\end{array}$ & $\begin{array}{l}\text { Binary } \\
\text { variables }\end{array}$ & $\begin{array}{l}\text { E(Benefits) } \\
€\end{array}$ & $\begin{array}{l}\text { CPU } \\
\text { s }\end{array}$ \\
\hline 31927 & 9915 & 5240 & 850.058 & 893 \\
\hline
\end{tabular}

Table 7: Stochastic Programming Indicators

\begin{tabular}{|c|c|}
\hline \multicolumn{2}{|c|}{ Monday, May 05, 2008} \\
\hline $\mathrm{RP}$ & $850.058 €$ \\
\hline EEV & $830.695 €$ \\
\hline VSS & $19.363 €$ \\
\hline
\end{tabular}

solved with CPLEX (CPLEX, 2008) (default options) using a SunFire X2200 with two dual core AMD Opteron 2222 processors at $3 \mathrm{GHz}$ and $32 \mathrm{~Gb}$ of RAM memory.

In order to test the two-stage stochastic model (32) a characterization of the market price $\lambda^{D}$ through a set of scenarios is required, where each scenario $\lambda^{D, s}=\left[\lambda_{1}^{D, s}, \ldots, \lambda_{24}^{D, s}\right]$ is composed by a set of 24 hourly market prices. A set of 25 scenarios has been used, obtained as the result of the application of a scenario reduction algorithm (Gröwe-Kuska et al, 2003) to the complete set of history data available from June 2007 to the day under study. A summary of the characteristics of the optimization problem and its solution is shown in Table 6. In Table 7 the usual stochastic programming indicators needed to evaluate the goodness of the stochastic approximation are reported (Birge and Louveaux, 1997). VSS, the measure of the advantage of using the stochastic programming model instead of the deterministic one, shows that is is possible to increase the expected benefits in $19.363 €(2.33 \%)$ by using the stochastic optimal solution.

The optimal unit commitment of thermal and CC units is shown in Fig. 6. The three states or configurations of the $\mathrm{CC}$ units are represented as white (state 0 ), gray (state $1, \mathcal{P}_{c}(1)$ ) and black (state $2, \mathcal{P}_{c}(2)$ ) hourly blocks. Notice how the operation of the CC units obey the minimum up time and the feasible transition rules expressed by Eq. (2) and Eq. (6) respectively. When startedup, both CC units stay in state 1 longer than the minimum on time $t_{t}^{o n}=2$ before switching to the state 2, where they remain for the rest of the optimization period.

Fig. 7 shows the aggregated economic dispatch of the three BCs (600MWh) by the thermal (white bars) and the CC (black bars) units. It can be observed that, depending on the period, the portfolio of BC is covered exclusively by the thermal units (periods 1,2,10,15,19 and 24), or by a combination of thermal and CC units (the rest of the periods).

The optimal bid functions (12) for the thermal and CC units are represented in Fig. 8 and Fig. 9 respectively, where $b_{i \ldots k}$ represents the value of $b_{i t}$ at the different periods $i$, and $b_{*}$ corresponds

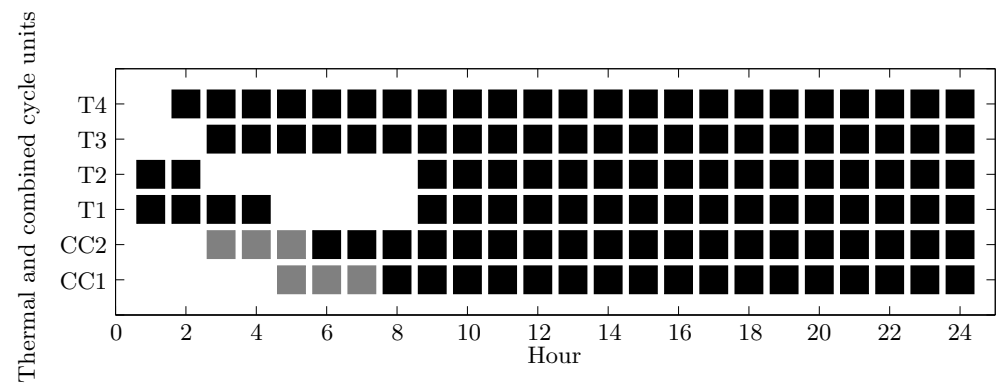

Figure 6: The unit commitment of thermal and CC units. 


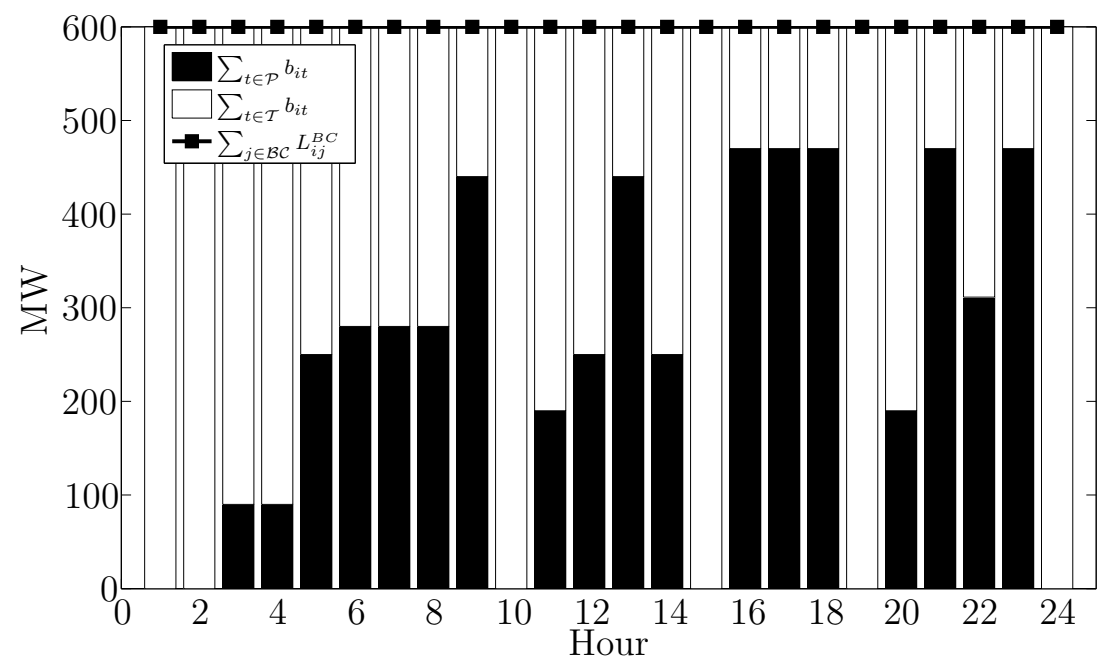

Figure 7: Management of the bilateral contracts $\left(\sum_{j \in \mathcal{B C}} L_{i j}^{B C}=600 \mathrm{MW}\right)$ between thermal and combined cycle units.
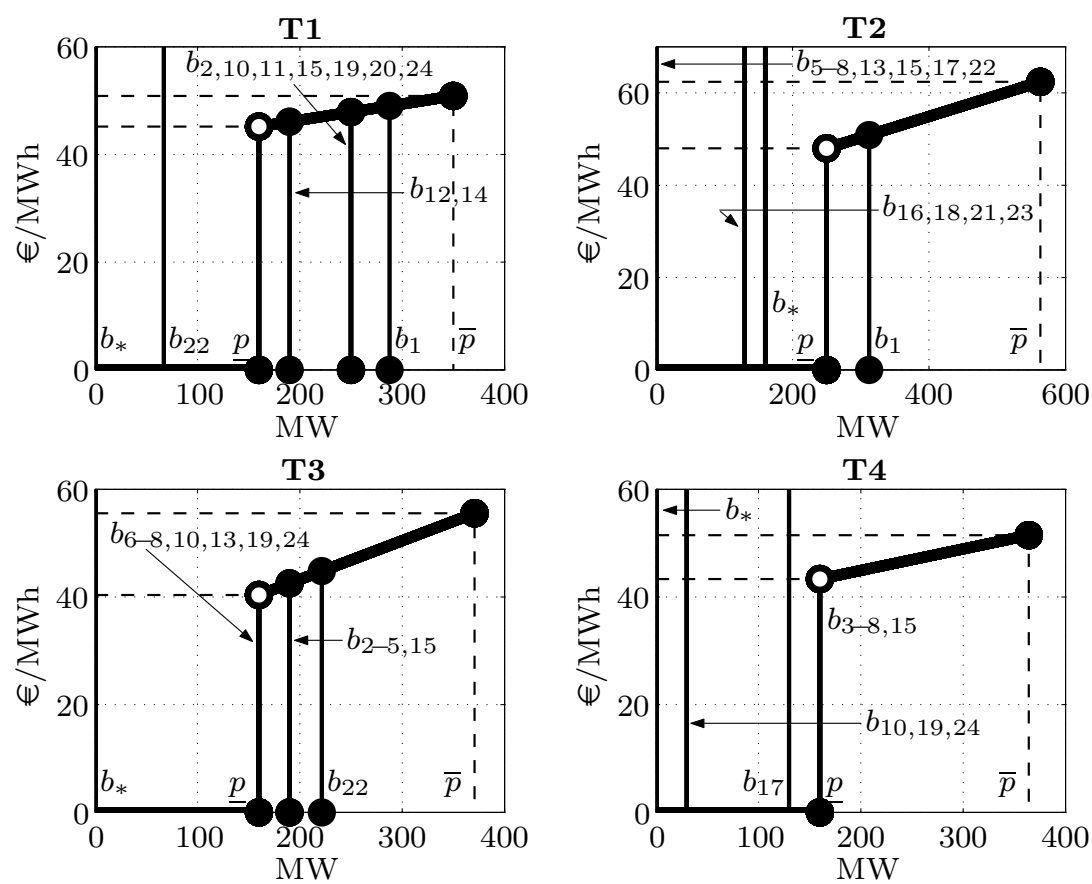

Figure 8: Representation of the optimal bid function $\lambda_{i t}^{B *}\left(p_{i t}^{B}, b_{i t}\right)$ of the thermal units. 

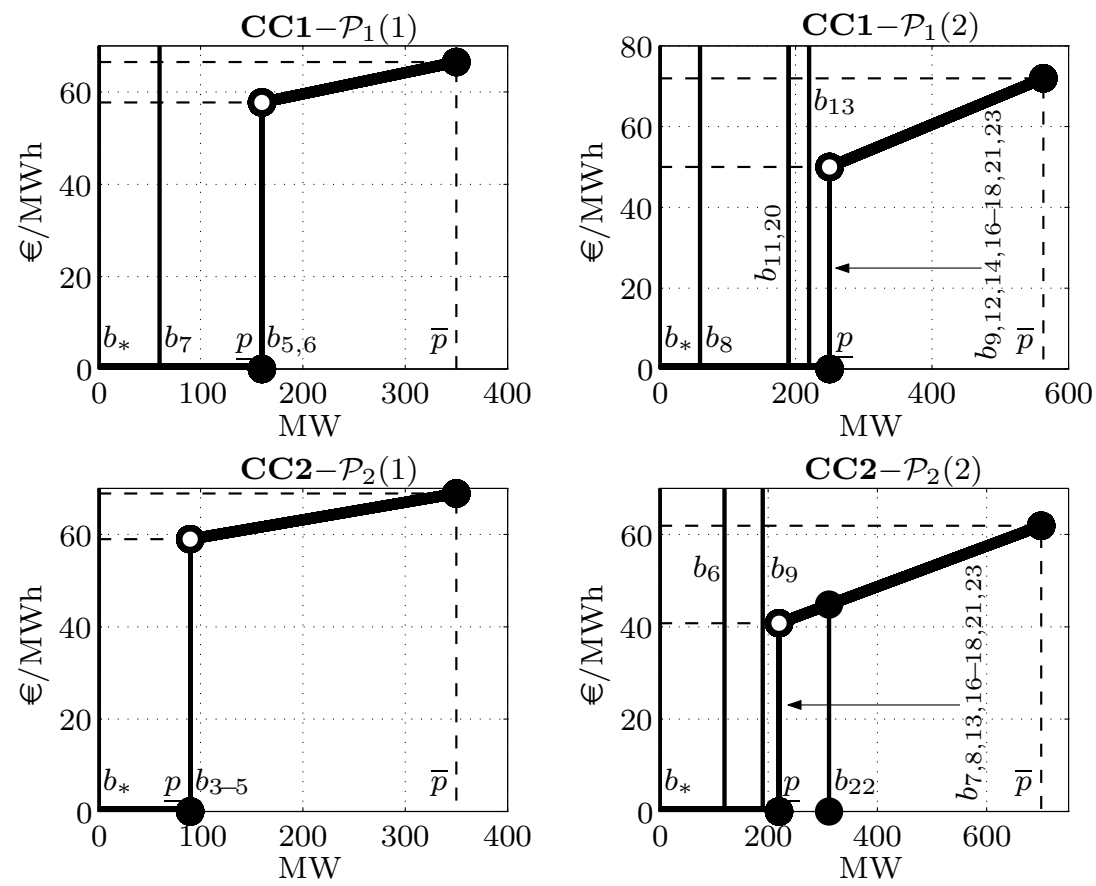

Figure 9: Representation of the optimal bid function $\lambda_{i t}^{B *}\left(p_{i t}^{B}, b_{i t}\right)$ of the CC units.

to the rest of periods not explicitly indicated. To help the understanding of this graphics, let us analyze the most simple case, the thermal unit T4, which is committed all the periods, except the first one. First, observe the piecewise discontinuous thick line, with a first block going from 0 to the minimum output $p$, and a second block between $p$ and $\bar{p}$, with a slope equal to the marginal cost of the thermal unit $\overline{2} c_{4}^{q}$. Both blocks correspond, respectively, to the two blocks defining the optimal bid function (12). After the development of Section 2.3 we know that this thick line represents the optimal bid function only in those periods where $b_{i t}=0$, (periods $i \in\{2,9,11-14,16,18,20-23\}$ for the thermal unit T4). Moreover, for those periods where $b_{i t}>0$, the optimal bid function corresponds to the part of the thick line between the auxiliary second vertical axis shown in Fig. 8 located at $b_{i t}$, and $\bar{p}_{t}$. In the case of the thermal unit T4, $b_{i, 4}=\underline{p}_{4}=160 \mathrm{MW}$ for periods $i \in\{3-8,15\}, b_{i, 4}=130 \mathrm{MW}$ for period $i=17$ and $b_{i, 4}=30 \mathrm{MW}$ for periods $i \in\{10,19,24\}$. Although $b_{i, 4} \leq \underline{p}_{4} \forall i$ in the case of the thermal unit T4, this could not be the case for other thermal units: see for instance the optimal bid function of thermal unit T3, where $b_{i, 3}=190 \mathrm{MW}$ $\forall i \in\{2-5,15\}$ and $b_{22,3}=221.5 \mathrm{MW}$, both values above the minimum generation $\underline{p}_{3}=160 \mathrm{MW}$. The optimal bid functions of the remaining thermal units of Fig. 8 can be interpreted in a similar way.

Let us now focus our attention on the optimal bid functions of the CC units (Fig. 9). First observe how each CC has two different sets of optimal bid functions, depending on the state of the $\mathrm{CC}$ unit at each period $i$. The $\mathrm{CC}$ unit 1 would send the optimal bid functions CC1- $\mathcal{P}_{1}(1)$ at periods 5,6 and 7, where this CC unit is in state 1 (gray blocks of Fig. 6), and the optimal bid functions CC1- $\mathcal{P}_{1}(2)$ at the rest of the periods (black blocks of Fig. 6). The same happens with the second CC unit, CC2. Please notice that the optimal bid function of each state of the same $\mathrm{CC}$ unit has its own slope, which corresponds to the marginal cost of each PU.

\section{Conclusions}

This study provides a procedure for a price-taker generation company operating under the most recent regulations of the Iberic Electricity Market to optimally manage a pool of thermal and combined cycle units. The proposed technique is built within the versatile decision framework provided by the stochastic programming methodology. A two-stage stochastic mixed-quadratic 
programming problem has been proposed to decide the optimal unit commitment and sale bid to the day-ahead market, and the optimal economic dispatch of the bilateral contracts for all the thermal and combined cycle units observing the MIBEL regulation. The objective of the producer is to maximize the expected profit from its involvement in the spot market. The most relevant contributions of this study include: (a) the integration of a new model for the combined cycle units with the thermal units and the bilateral contracts covering and (b) a new modelization of the optimal sale bid for both thermal and combined cycle units, with respect to the dispatched energy of the bilateral contracts. The set of scenarios representing the uncertainty of the spot prices has been built by applying reduction techniques to the tree obtained from the actual data of the MIBEL system. The model was implemented and solved with commercial optimization packages and tested with real data of a Spanish generation company and market prices. The results of the computational experiments show the validity of the presented model and its applicability to real problems.

\section{Acknowledgements}

We thank Dr. Narcis Nabona, Dr. Adela Pagès, and Mr. Matteo Tesser from the Group on Numerical Optimization and Modelling at the Technical University of Catalonia; Mr. Antonio Canoyra from Union Fenosa, and Ms. Teresa Sanz and Mr. Emilio Cortes, from Gas Natural, for their valuable comments and suggestions. We also express our gratitude to Ms. Glòria Casanellas for her support in the elaboration of several parts of this paper.

\section{References}

Anderson EJ, Philpott AB (2002) Optimal offer construction in electricity markets. Mathematics of Operations Research 27(1):82-100

Anderson EJ, Philpott AB (2003) Using supply functions for offering market generation into an electricity market. Operations Research 50(3):477-489

BELPEX (2008) Belgium virtual power plant auctions. http://www.belpexvpp.be/. Accessed 30 september 2008

Birge JR, Louveaux F (1997) Introduction to Stochastic Programming. New York: Springer-Verlag

Bjelogrlic MR (2000) Inclusion of combined cycle plants into optimal resource scheduling. Proc 2000 IEEE PES Summer Meeting pp 189-195

CESUR (2008) Spain cesur bilateral contracts auctions. http://www.subastascesur.omel.es/. Accessed 30 september 2008

Conejo NFJ A J, Arroyo JM (2002) Price-taker bidding strategy under price uncertainty. IEEE Trans Power Syst 17(4):1081-1088

CPLEX (2008) CPLEX optimization subroutine library guide and reference. Version 11.0. CPLEX Division, ILOG Inc., Incline Village, NV, USA

EDF (2008) Electricité de france capacity auctions. http://capacityauctions.edf.fr/. Accessed 30 september 2008

Fourer R, Gay DM, Kernighan BW (2003) AMPL: A modeling language for mathematical programming, 2nd edn. CA: Brooks/Cole-Thomson Learning

Gountis VP, Bakirtzis AG (2004) Bidding strategies for electricity producers in a competitive electricity marketplace. IEEE Trans Power Syst 19:356-365

Gröwe-Kuska N, Heitsch H, Römisch W (2003) Scenario reduction and scenario tree construction for power management problems. Proc IEEE PowerTech 3, bologna, Italy 
Li T, Shahidehpour M (2005) Price-based unit commitment: A case of lagrangian relaxation versus mixed integer programming. IEEE Trans Power Syst 20(4):2015-2025

Lu B, Shahidehpour M (2004) Short-term scheduling of combined cycle units. IEEE Trans Power Syst 19(3):1616-1625

MEFF (2008) Spain virtual power plant auctions. https://www.subasta-epe.com/. Accessed 30 september 2008

Nabona N, Pages A (2007) A three-stage short-term electric power planning procedure for a generation company in a liberalized market. International Journal of Electric Power Systems 29(5):408421

Neame P, Philpott AB, Pritchard G (2003) Offer stack optimisation in electricity pool markets. Operations Research 51(3):397-408

Ni E, Luh PB, Rourke S (2004) Optimal integrated generation bidding and schedulling with risk management under a deregulated power market. IEEE Trans Power Syst 19(1):600-1088

Nowak M, Schultz R, Westphalen M (2005) A stochastic integer programming model for incorporating day-ahead trading of electricity into hydro-thermal unit commitment. Optimization and Engineering 6:163-176

OMEL (2008) Market operator of the electricity market of mainland spain. http://www.omel.es/. Accessed 30 september 2008

Shresta GB, Kai S, Goel L (2004) An efficient stochastic self-scheduling technique for power producers in the deregulated power market. Electr Power Syst Res 71:91-98

Triki C, Beraldi P, Gross G (2005) Optimal capacity allocation in multi-auction electricity markets under uncertainty. Computers \& Operations Research 32(2):201-217 